\title{
Changes of Macular Vessel Density in Primary Open Angle Glaucoma Nesma Sayed Mohammed
}

Department of Ophthalmology, Faculty of Medicine, Al-Azhar University, Cairo, Egypt

Corresponding author: Nesma Sayed Mohammed, Mobile: (+20) 01014852522, E-Mail: nesma7@live.com

\begin{abstract}
Background: Glaucoma is a known cause of irreversible blindness worldwide. Several techniques are used for its diagnosis and follow-up. Optical coherence tomography angiography (OCTA) is new technology that provides a quantitative assessment of the microcirculation of the retina and optic nerve in a rapid, noninvasive method.

Objective: To evaluate the changes at the macular vessel density (MVD) in primary open glaucoma patients (POAG) to correlate these changes with the changes of both ganglion cell complex (GCC) thickness and retinal nerve fiber layer (RNFL) thickness. Patients and Methods: Fifty healthy participants and fifty primary open angle glaucoma (POAG) subjects underwent visual field analysis, measurement of the whole image MVD, measurement of the average (RNFL) thickness, and measurement of the average thickness of (GCC). The area under the curve (AUC) was assessed for each parameter to differentiate POAG from healthy eyes.

Results: MVD was lower in POAG than in the control group. The glaucomatous group showed that MVD was 21.18 \pm 2.6 at the fovea, while it was $39.5 \pm 4.71$ at the parafovea. There was a statistically significant positive correlation between MVD (foveal and parafoveal (especially at the superior quadrant) and the mean deviation (MD) (R+ve, $\mathrm{P}<$ $0.05)$. There was a statistically significant positive correlation between MVD and structural parameters. The ROC curves showed that the cut-off points were $<28.2$ and $<48$ for MVD at the fovea and parafoveal area respectively, at the superficial vascular plexus with $100 \%$ sensitivity \& $100 \%$ specificity between the controls and POAG eyes.

Conclusions: MVD was reduced in cases with POAG compared to the control group. The assessment of macular vessel density by OCTA gave us good diagnostic abilities for diagnosis and management of glaucoma.

Keywords: Macular vessel density, Ganglion cell complex, Optical coherence tomography angiography, Primary open angle glaucoma.
\end{abstract}

\section{INTRODUCTION}

Primary open-angle glaucoma (POAG) is a chronic progressive optic neuropathy that ultimately results in corresponding visual field (VF) defects ${ }^{(\mathbf{1})}$. The main risk factor is ocular hypertension (IOP) ${ }^{(2)}$. It is characterized by a damage of the retinal ganglion cells (RGCs) and the retinal nerve fibers (RNFs), which comprises RGCs axons. Their measurement could provide important information for the detection and evaluation of glaucoma. Though the glaucomatous damage is believed to involve the RNFs at first, but some authors have found that macular involvement can occur primarily before structural or functional loss appears, especially at the level of superficial capillary plexus (SCP), with relative sparing of the deep capillary plexus (DCP) ${ }^{(3)}$. Though the pathogenesis of glaucoma is still unclear, the vascular component of POAG has recently gained more interest. Optical coherence tomography angiography (OCTA) has been introduced as a noninvasive and reproducible tool to provide a qualitative and quantitative assessment of the microvasculature of the different retinal layers ${ }^{(4)}$.

The evaluation of the vasculature within the optic nerve, the periventricular region, the superficial retina, and the peritoneal choroid in glaucoma can be determined using optical coherence tomography angiography (OCTA). The assessment of superficial retinal vessel plexus vessel density aids in diagnosis and detection of glaucoma progression like the assessment of the peripapillary retinal nerve fiber layer (RNFL) thickness. Furthermore, decreased peripapillary vessel density of the intact hemiretina or unaffected eye of glaucomatous eyes suggests that these vascular changes can occur prior to detectable visual field damage ${ }^{(5)}$. Hence, it was the aim of this study to evaluate macular vessel density (MVD) in POAG patients.

The aim of the present work was to evaluate the changes at the macular vessel density (MVD) in primary open glaucoma patients (POAG) to correlate these changes with the changes of both ganglion cell complex (GCC) thickness and retinal nerve fiber layer (RNFL) thickness, and to evaluate the diagnostic accuracy of measuring these vascular parameters.

\section{PATIENTS AND METHODS}

This study was designed as a comparative prospective cross-sectional study. Patients were recruited from the Department of Ophthalmology, AlZahraa University Hospital.

\section{Ethical approval:}

An informed written consent was obtained from each participant after receiving a full explanation about the study. Besides, an approval was obtained from the Ethics Board of Al-Azhar University Committee. All procedures were conducted in accordance with the Helsinki Declaration guidelines.

This study included two groups; control group (50 eyes of 25 persons) and POAG group (50 eyes of 25 persons). Inclusion criteria for the control group included no family history of glaucoma, intraocular pressure less than (IOP) $21 \mathrm{mmHg}$, open angles on gonioscopy, 
normal anterior and posterior segment on slit-lamp examination, no glaucomatous optic disc, no RNFL defects, and no glaucomatous VF defects.

Inclusion criteria for the glaucomatous group included an open iridocorneal angle on gonioscopy, BCVA better than 20/40, IOP > $21 \mathrm{mmHg}$, glaucomatous optic disc changes (Neuroretinal rim thinning, rim notching or RNFL defect), reliable and repeatable glaucomatous visual field defect and a refractive error between -3 and 3 diopters.

Exclusion criteria: Presence of retinal or macular pathology, any media opacities, and any systemic or neurological conditions that could produce VF defects. Patients unable to perform reliable VF testing or with poor quality OCTA, including segmentation errors, were also excluded.

All participants underwent a comprehensive ocular examination, which included corrected distance visual acuity measurement, slit-lamp biomicroscopy, Goldmann applanation, tonometry, gonioscopy, fundus examination, visual field analysis, OCT and OCTA scanning.

\section{Humphrey visual field test:}

VF test was performed using the Swedish Interactive Threshold Algorithm (SITA) Standard strategy program 24-2 of the Humphrey Field Analyzer II (Carl Zeiss Meditec, Jena, Germany). Fixation losses $20 \%$, false positives, and false negatives $33 \%$ were established as reliability criteria. A normal VF was defined as a pattern standard deviation (PSD) within the 95\% confidence limits and a Glaucoma VF test within normal limits. Glaucomatous VF defects were defined as, a cluster of 3 points with $\mathrm{P}<0.05$ on the pattern deviation map in at least one hemifield, including 1 point with $\mathrm{P}<0.01$, a PSD of $\mathrm{P}<0.05$, or glaucoma VF test result outside the normal limits.

\section{Structural parameters and vessel density:}

Optic nerve head $(\mathrm{ONH})$ and macular imaging were also performed using the (Avanti; Optovue, Inc., Fremont, CA, USA) OCT system on the same day as OCTA imaging and by the same operator. It uses a light source with center wavelength of $840 \mathrm{~nm}$ and it has an A -scan rate of $70-\mathrm{kHz}$. The ONH scanning protocol was used for measuring the RNFL thickness and the macular ganglion cell (GCC) scanning protocol was used to measure the GCC thickness. The GCC scanning protocol consists of a $7 \times 7 \mathrm{~mm}^{2}$ raster scan consisting of 1 horizontal B scan of 933 A-scans and 15 vertical B scans of 933 A-scans per B-scan. This protocol measures retinal thickness from the internal limiting membrane (ILM) to the inner plexiform layer (IPL) posterior boundary obtained from the raster scans and GCC thickness measurements consist of the ganglion cell layer, IPL and RNFL. The mean GCC thickness measurements were used for the analysis. Images that were accepted were those of signal strength index (SSI) $\geq 37$, and without any segmentation failure or artifacts.

\section{OCT Angiography Image Acquisition and Processing:}

All subjects underwent OCTA imaging with a commercially available OCTA system (Avanti; Optovue, Angiovue, Inc., Fremont, CA, USA) that is incorporated in the Avanti OCT system. The Angiovue imaging system provides a noninvasive method for segmenting the retinal vasculature at the capillary level. It utilizes the Split-Spectrum Amplitude-Decorrelation Angiography (SSADA) algorithm. The Angiovue characterizes vascular information at various userdefined retinal layers qualitatively as a vessel density map and color-coded vessel area density.

For the present study, MVD measurements were calculated from $3 \times 3 \mathrm{~mm}$ scans centered on the fovea at the SCP. For both measurements region segmentation was performed using the OCT intensity B-scans. Macular superficial vessel density measurements were calculated in a slab from ILM to the posterior boundary of the inner plexiform layer (IPL).

Vessel length density, defined as the total length of perfused vasculature per unit area in the region of measurement. Poor quality scans were excluded from the analysis if the signal strength index (SSI) when was lower than 50 , or there were floaters, projection or motion artifacts.

\section{Statistical analysis}

Analysis of data was performed using software MedCalc v. 19. Description of variables was presented where the description of quantitative variables was in the form of mean, standard deviation (SD), minimum and maximum. Description of qualitative variables was in the form of numbers (No.) and percent (\%). Data were explored using Kolmogorov-Smirnov test of normality. The results of Kolmogorov-Smirnov test indicated that most of data were normally distributed (parametric data) so parametric tests were used for most of the comparisons. Comparison between quantitative variables was carried out by one-way analysis of variance (ANOVA), which was used to test the difference between the means of several subgroups of a variable. Binary correlation was carried out by Pearson correlation test. Results were expressed in the form of correlation coefficient (R) and P-values. The diagnostic performance of a test or the accuracy of a test to discriminate diseased cases from normal cases is evaluated using Receiver Operating Characteristic (ROC) curve analysis. ROC curves can also be used to compare the diagnostic performance of two or more laboratory or diagnostic tests. $\mathrm{P}$ value $\leq 0.05$ was considered significant.

\section{RESULTS}

One hundred eyes of 50 patients ( 35 males and 15 females) were enrolled in this study. Their mean age was $53.50 \pm 9.37$ ranged from $40-81$ years. There was a statistically significant difference between the two groups regarding BCVA, IOP and C/D ratio (Table 1). 
Table (1): Comparing ocular measurements between case and control by ANOVA test

\begin{tabular}{|l|c|c|c|c|c|}
\hline \multirow{2}{*}{ Ocular measurement } & \multicolumn{2}{|c|}{ Case (POAG) } & \multicolumn{2}{c|}{ Control } & \multirow{2}{*}{ P. value } \\
\cline { 2 - 5 } & Mean & SD & Mean & SD & \multirow{2}{*}{$0.046^{*}$} \\
\hline BCVA & 0.86 & 0.21 & 0.93 & 0.09 & $<0.001^{*}$ \\
\hline C/D ratio & 0.63 & 0.16 & 0.28 & 0.11 & $<0.001^{*}$ \\
\hline IOP $(\mathrm{mmHg})$ & 19.02 & 2.60 & 12.88 & 1.94 & \\
\hline
\end{tabular}

$* \mathrm{P} \leq 0.05$ is considered significant

The changes of functional parameters, structural parameters and macular vessel density:

There were statistically significant differences in all measurements among the groups, including the functional parameters (MD and PSD), structural parameters (average RNFL thickness and GCC thickness) and MVD (foveal and average parafoveal) $(\mathrm{p}<0.01)$. In the en face OCTA image of the superficial vascular complex $(\mathrm{SCP})$, there were centripetally branching vessels terminating in the central foveal avascular zone (FAZ).

The branching vessels were observably denser in normal eyes than those with glaucoma. We found that the average MVD (at the fovea) was $21.18 \pm 2.6$ in the glaucomatous group and $33.30 \pm 2.35$ in the control group. While MVD (at the parafovea) was $39.5 \pm 4.71$ in the glaucomatous group and $56.48 \pm 3.84$ in the control group (Table 2).

Table (2): Comparing the visual field, OCT \& OCTA variables between case and control

\begin{tabular}{|l|c|c|c|c|c|}
\hline \multirow{2}{*}{ Visual field change } & \multicolumn{2}{|c|}{ Case (POAG) } & \multicolumn{2}{c|}{ Control } & \multirow{2}{*}{ P value } \\
\cline { 2 - 5 } & Mean & SD & Mean & SD & \multirow{2}{*}{} \\
\hline MD & -6.04 & 4.53 & -0.89 & 0.50 & $<0.001^{*}$ \\
\hline PSD & 5.05 & 1.92 & 1.33 & 0.43 & $<0.001^{*}$ \\
\hline RNFL & 86.07 & 12.60 & 101.52 & 3.87 & $<0.001^{*}$ \\
\hline GCC & 87.27 & 11.17 & 94.84 & 2.38 & $<0.001^{*}$ \\
\hline Foveal VD & 21.18 & 2.60 & 33.30 & 2.35 & $<0.001^{*}$ \\
\hline Parafoveal VD & 39.50 & 4.71 & 56.48 & 3.84 & $<0.001^{*}$ \\
\hline
\end{tabular}

\section{Correlation of macular vessel density with structural and functional tests}

There was a statistically significant positive correlation between MVD (fovea and parafovea (especially at the superior quadrant) and the mean deviation ( $\mathrm{R}+\mathrm{ve}, \mathrm{P}<0.05)$. On the other hand, there was a statistically significant negative correlation between PSD and the MVD at both the fovea and parafovea, especially at the inferior and nasal quadrants (R-ve, $\mathrm{P}<0.05)$ (Table 3).

Table (3): Pearson correlation between VF parameters and MVD in POAG group

\begin{tabular}{|l|c|c|c|c|}
\hline \multirow{2}{*}{$\begin{array}{c}\text { Foveal VD } \\
\text { (POAG group) }\end{array}$} & \multicolumn{2}{|c|}{ MD } & \multicolumn{2}{c|}{ PSD } \\
\cline { 2 - 5 } & $\mathrm{R}$ & $\mathrm{P}$ value & $\mathrm{R}$ & P value \\
\hline Superficial & 0.272 & 0.0711 & -0.931 & $<0.0001^{*}$ \\
\hline Superficial parafoveal & 0.377 & $0.0108^{*}$ & -0.545 & $0.0001^{*}$ \\
\hline Superior & 0.316 & $0.0343^{*}$ & -0.266 & 0.0769 \\
\hline Inferior & 0.459 & $0.0015^{*}$ & -0.356 & $0.0165^{*}$ \\
\hline Nasal & 0.131 & 0.3900 & -0.505 & $0.0004^{*}$ \\
\hline Temporal & 0.133 & 0.3835 & -0.014 & 0.9274 \\
\hline
\end{tabular}

$* \mathrm{P} \leq 0.05$ is considered significant

There was a statistically significant positive correlation between RNFL thickness and superficial parafoveal $\mathrm{VD}$, superior and inferior foveal $\mathrm{VD}(\mathrm{R}+\mathrm{ve}, \mathrm{P}<0.05)$. Also, there was statistically significant positive correlations between GCC thickness and superficial foveal, parafroveal, superior, inferior, temporal foveal VD (R+ve, $\mathrm{P}<0.05)$ (Table 4). 
Table (4): Pearson correlation between RNFL, GCC and foveal VD in POAG group

\begin{tabular}{|l|c|c|c|c|}
\hline \multirow{2}{*}{$\begin{array}{c}\text { Foveal VD } \\
\text { (POAG group) }\end{array}$} & \multicolumn{2}{|c|}{ RNFL $(\mu \mathrm{m})$} & \multicolumn{2}{c|}{ GCC $(\mu \mathrm{m})$} \\
\cline { 2 - 5 } & $\mathrm{R}$ & P.value & $\mathrm{R}$ & P.value \\
\hline Superficial & 0.245 & 0.1045 & 0.348 & $0.0191^{*}$ \\
\hline Superficial parafoveal & 0.499 & $0.0005^{*}$ & 0.511 & $0.0003^{*}$ \\
\hline Superior & 0.382 & $0.0097^{*}$ & 0.579 & $<0.0001^{*}$ \\
\hline Inferior & 0.362 & $0.0145^{*}$ & 0.416 & $0.0045^{*}$ \\
\hline Nasal & 0.171 & 0.2625 & 0.154 & 0.3128 \\
\hline Temporal & 0.259 & 0.0858 & 0.444 & $0.0022^{*}$ \\
\hline
\end{tabular}

RNFL: Retinal nerve fiber layer, GCC: Ganglion cell complex * $\mathrm{P}<0.05$ is considered significant

The ROC curves showed that the cut-off points were $<28.2$ and $<48$ for VD at the fovea and parafovea respectively at the SCP with $100 \%$ sensitivity and $100 \%$ specificity between the control and POAG eyes. The area under the ROC curve analysis was 1 for both (Table 5).

Table (5): Predictive value of MVD for POAG (ROC) analysis

\begin{tabular}{|l|c|c|c|c|c|}
\hline \multirow{2}{*}{ Foveal VD } & \multicolumn{5}{c|}{ POAG } \\
\cline { 2 - 6 } & AUC & Cut off & Sens\% & Spec\% & P.value \\
\hline Superficial & 1 & $\leq 28.2$ & 100 & 100 & $<0.0001^{*}$ \\
\hline Superficial parafoveal & 1 & $\leq 48$ & 100 & 100 & $<0.0001^{*}$ \\
\hline Superior & 0.892 & $\leq 50$ & 84.44 & 96 & $<0.0001^{*}$ \\
\hline Inferior & 1 & $\leq 49$ & 100 & 100 & $<0.0001^{*}$ \\
\hline Nasal & 0.961 & $\leq 47$ & 95.56 & 100 & $<0.0001^{*}$ \\
\hline Temporal & 0.857 & $\leq 49$ & 84.44 & 100 & $<0.0001^{*}$ \\
\hline
\end{tabular}

POAG: Primary open-angle glaucoma, AUC: Area under curve, Sens: Sensitivity, Spec: Specificity

$* \mathrm{P}<0.05$ is considered significant

\section{DISCUSSION}

In the present study, we compared functional parameters (MD and PSD), structural OCT parameters (GCC, and RFNL thickness) and macular VD across patients with POAG, and control subjects.

In our study we found that, GCC and RNFL were thinner in the glaucomatous eyes compared to the control subjects. This agrees with what has already been demonstrated that RNFL thickness reduction occurs in glaucoma especially measuring the angular width of localized RNFL defect is also a viable method for determining the severity of visual field defect ${ }^{(6,7)}$.

The relationship between ocular blood flow changes and POAG was controversy. Some consider it as a major pathogenic factor ${ }^{(8)}$. Several instruments have been used to detect the changes of ocular blood flow in glaucoma (e.g. fluorescein and indocyanine green angiography ${ }^{(9)}$ and confocal scanning laser Doppler flowmetry) ${ }^{(10)}$. Although welcomed with enthusiasm, none of these eventually broke into clinical practice due to several limitations (e.g. reliability, invasiveness, accuracy, and precision) ${ }^{(11)}$.

OCTA has several advantages over previous devices since it is a non-invasive, reliable, depthresolved, user-friendly technique has already started to expand our knowledge base regarding the role of ocular blood flow in glaucoma ${ }^{(\mathbf{1 2})}$. OCTA divides the retinal capillaries into radial peripapillary, superficial, and deep capillary plexuses. Superficial retinal capillaries (SRCs) have a centripetal pattern with many interconnections to each other (forming a honeycomb pattern) and to more superficial and deeper vascular plexuses ${ }^{(13)}$. SRCs feed and match RGCs and therefore they interrupt in correspondence of the fovea forming a capillary-free zone, named foveal avascular zone ${ }^{(14)}$.

In our study, we found a statistically significant lower mean MVD in the POAG group than in the control group. This is consistent with $\mathbf{L i}$ et al. ${ }^{(15)}$ and Lommatzsch et al. ${ }^{(16)}$ where they found decreased MVD in both the superficial layer and the deep layer in glaucomatous eyes. The present study is in contrast with the study of Triolo et al. ${ }^{(\mathbf{1 7})}$. They conducted their study on 40 controls, 40 glaucoma suspects, and 40 glaucoma patients. Where they did not find any difference in the macular vessel density in all groups. The significant differences were for structural parameters not for vascular parameters.

In addition, we found correlations between the vascular parameters and both the structural and functional ones. MVD was significantly correlated with structural variables (i.e., GCC thickness, RNFL thickness) and also with VF indices (MD and PSD). This 
is in agreement with Almbsut $\boldsymbol{e t} \boldsymbol{a l} .{ }^{(18)}$ who stated that the vessel density was significantly correlated with MD $(\mathrm{r}=0.404, \mathrm{p}=0.001)$, RNFL thickness $(\mathrm{r}=0.465, \mathrm{p}<$ $0.001)$ and GCC thickness.

In the present study, we have focused on the superficial vascular plexuses since glaucoma is a disease of RGCs and their axons (i.e., RNFs), which are nourished by the inner plexuses. Moreover, deep capillary plexus may be affected by projection artifacts. This is in agreement with Akil et al. ${ }^{(19)}$ who found a statistically significant decrease of the MVD, at the superficial and deep retinal layer, between glaucomatous eyes and control. They reported that although the two layers were seen as affected, an increasingly predictable and extreme decrease in vessel density was assessed in the superficial retinal layer than in the deep one. Also, Takusagawa et al. (20) found that glaucoma preferentially affects perfusion in the SCP in the macula more than the DCP.

The ROC curves showed that the cut-off points were $<28.2$ and $<48$ for VD at the fovea and parafoveal area at the superficial plexus with $100 \%$ sensitivity and $100 \%$ specificity between the controls and POAG eyes. This is in agreement with Almbsut $\boldsymbol{e t}$ al. ${ }^{\left({ }^{(18)}\right.}$ and Akil $\boldsymbol{e t}$ al. ${ }^{(19)}$ where both showed that the area under the ROC curve analysis had a high diagnostic accuracy for differentiating POAG from the control.

Disagreement among studies could be related to differences in the study population (e.g., glaucoma severity), study design, OCTA technical aspects e.g. the vessel perfusion density, calculation of algorithm, or image quality. Furthermore, the difference could be attributed to different SCP segmentation methods. From the above-mentioned results of the current study, we found that measuring MVD using OCTA is considered a good diagnostic tool for the diagnosis and management of POAG.

\section{CONCLUSION}

MVD measurements were lower in POAG in relation to the control. They were better correlated with both structural and functional parameters. This demonstrates the importance of measuring the retinal microcirculation parameters in the macular area in cases of POAG. The inclusion of OCTA in glaucoma diagnostics may improve our understanding of ocular blood flow changes in glaucoma.

\section{REFERENCES}

1. Weinreb R, Leung C, Crowston J et al. (2016): Primary open angle glaucoma. Nat Rev Dis Primers, 2: 160-67.

2. Soliman T, Gad E, Selim S (2019): Ganglion cell analysis versus retinal nerve fiber layer thickness in glaucoma diagnosis. J Egypt Ophthalmol Soc., 112: 122-9.

3. Kim J, Kim Y, Baek S et al. (2020): Topographic correlation between macular superficial microvessel density and ganglion cell-inner plexiform layer thickness in glaucoma-suspect and early normal-tension glaucoma. Br. J. Ophthalmol., 104: 104-109.

4. Rabiolo A, Carnevali A, Bandello F et al. (2016): Optical coherence tomography angiography: evolution or revolution? Expert Rev Ophthalmol., 11: 243-245.

5. Aghsaei Fard M, Ritch R (2020): Optical coherence tomography angiography in glaucoma. Ann Transl Med., 8 (18): 1204.

6. Banitt M, Ventura L, Feuer W et al. (2013): Progressive loss of retinal ganglion cell function precedes structural loss by several years in glaucoma suspects. Invest Ophthalmol Vis Sci., 54: 2346-2352.

7. Chen A, Lai I, Cho W et al. (2020): Defective angles of localized retinal nerve fiber layer reflect the severity of visual field defect- a cross-sectional analysis. BMC Ophthalmol., 20 (1): 141-145.

8. Flammer J, Mozaffarieh M (2007): What is the present pathogenetic concept of glaucomatous optic neuropathy? Surv Ophthalmol., 52 (2): 162-173.

9. Raitta C, Sarmela T (1970): Fluorescein angiography of the optic disc and the peripapillary area in chronic glaucoma. Acta Ophthalmol., 48: 303-308.

10. Logan J, Rankin S, Jackson A (2004): Retinal blood flow measurements and neuroretinal rim damage in glaucoma. $\mathrm{Br}$ J Ophthalmol., 88: 1049-1054.

11. Schuman J (2015): Measuring blood flow: so what? JAMA Ophthalmol., 133: 1052-1053.

12. Dastiridou A, Chopra V (2018): Potential applications of optical coherence tomography angiography in glaucoma. Curr Opin Ophthalmol., 29 (3): 226-233.

13. Campbell J, Zhang M, Hwang T et al. (2017): Detailed vascular anatomy of the human retina by projectionresolved optical coherence tomography angiography. Sci Rep., 7: 42201-7.

14. Cicinelli M, Carnevali A, Rabiolo A et al. (2017): Clinical spectrum of macular-foveal capillaries evaluated with optical coherence tomography angiography. Retina, 37: 436-443.

15. Li Z, Xu Z, Liu Qiang C et al. (2020): Comparisons of retinal vessel density and glaucomatous parameters in optical coherence tomography angiography. PLOS ONE, 15: 0234816.

16. Lommatzsch $\mathrm{C}$, Rothaus K, Koch J et al. (2018): OCTA vessel density changes in the macular zone in glaucomatous eyes. Graefes Arch Clin Exp Ophthalmol., 256 (8): 14991508.

17. Triolo G, Rabiolo A, Shemonski N et al. (2017): Optical coherence tomography angiography macular and peripapillary vessel perfusion density in healthy subjects, glaucoma suspects, and glaucoma patients. Invest Ophthalmol Vis Sci., 58: 5713- 5722.

18. Almbsut M, Abdelhameed A, Sabry D et al. (2020): Evaluation of Macular Vessels Density Changes in Patients with Primary Open Angle Glaucoma by Swept-source Optical Coherence Tomography Angiography. ARC Journal of Ophthalmology, 5 (1): 6-13.

19. Akil H, Chopra V, Al-sheikh M et al. (2018): Sweptsource OCT angiography imaging of the macular capillary network in glaucoma. Br J Ophthalmol., 102: 515-519.

20. Takusagawa H, Liu L, Ma K et al. (2017): Projectionresolved optical coherence tomography angiography of macular retinal circulation in glaucoma. Ophthalmology, 124: 1589-1599. 